Präv Gesundheitsf 2009 · 4:5-6 DOI 10.1007/s11553-008-0156-7

Online publiziert: 17. Januar 2009

๑) Springer Medizin Verlag 2009

\author{
H. Ohlbrecht \\ Lehrstuhl für Soziologie der Rehabilitation, berufliche Rehabilitation \\ und Rehabilitationsrecht, Philosophische Fakultät IV, \\ Institut für Rehabilitationswissenschaften, Humboldt-Universität zu Berlin
}

\title{
Editorial
}

Eine Gemeinsamkeit der Beiträge in diesem Heft aus so unterschiedlichen Bereichen wie etwa der Notaufnahme, der Sturzprophylaxe in Pflegeeinrichtungen, der Erschließung von Ressourcen in der gemeindeorientierten Arbeit von Hausarztpraxen, der betrieblichen Gesundheitsförderung, der Raucherprävention bei Jugendlichen usw. liegt in der für die Gesundheitsversorgung immer bedeutsameren Perspektive von Prävention und Gesundheitsförderung. In diesen Rahmen fügt sich auch ein theoretischer Beitrag zur Berücksichtigung der in der Gesundheitsforschung vernachlässigten Dimension von Emotionen ein.

Im Einzelnen berichten die vorliegenden Beiträge über folgende Themen: der Beitrag von Ohlbrecht, Bartel, v. Kardorff und Streibelt reagiert auf die aus der Praxis berichtete und auch international belegte Zunahme von Gewalthandlungen in der klinischen Notaufnahme. Dies wirkt sich nicht nur auf Arbeitszufriedenheit und Belastungen des Pflegepersonals aus, sondern erzeugt zusätzliche Kosten und ist möglicherweise mit Qualitätseinbußen verbunden. So muss Gewalt in verschiedenen Ausprägungen, wie physischer, psychischer und/oder verbaler Art, auch hinsichtlich der Interaktionsdynamik, der Bedingungen und Anlässe für Gewalthandlungen im Setting der Notaufnahme betrachtet werden, um diese Erkenntnisse für Gesundheitsförderung und für die Prävention nutzen zu können.

Besonders in stationären Pflegeeinrichtungen stellen die Sturzrisiken eine Herausforderung für infrastrukturelle und klientenbezogene Präventionsanstrengungen dar. Anhand eines deterministischen systemdynamischen Sturz- risikofaktorenmodells können die Autoren Zeller, Needham, Betschon, Hügel, Bischofberger und Hürni zeigen, dass die Sturzwahrscheinlichkeit einschätzbar ist und hier Ursache-Wirkungs-Zusammenhänge wie z. B. Ungleichgewicht zwischen Gehanforderungen und Gehfähigkeiten etc. direkt ableitbar sind, die für die Praxis wichtige Handlungsempfehlungen darstellen und eine gute Ergänzung der von der vom DNQB [1] erarbeiteten Qualitätsniveaus für diesen Bereich darstellen.

In ihrem Beitrag wendet sich Ulla Franken dem Schlüsselfaktor Emotion für ein umfassendes Verständnis der biopsychosozialen Theorie von Gesundheit und Krankheit zu. Emotionen können als „missing link“ in den Gesundheitswissenschaften betrachtet werden. Hier zeigt die Autorin auf, dass gerade die Berücksichtigung der Erkenntnisse der Emotionsforschung die verbreitete Risikofaktorenzentrierung überwinden hilft und eine breitenwirksame, am Konzept Salutogenese orientierte Gesundheitsförderung ermöglicht und damit zu einer erweiterten theoretischen aber auch für die Praxis bedeutsamen Wahrnehmungsdimension beiträgt.

Eine Bestandsaufnahme zu den hausärztlichen Erfahrungen und zu den Aspekten der Nutzung kommunaler Ressourcen bei chronischen Erkrankungen stellt die Studie von Bölter, Natanzon, Miksch, Joos, Rosemann, Szecsenyi, Götz dar. Chronische Krankheiten nehmen weltweit $\mathrm{zu}$ und stellen innerhalb des medizinischen Versorgungssystems eine große Herausforderung dar. Die Autoren arbeiten heraus, dass die befragten Hausärzte Ressourcen hinsichtlich der Prävention sehen und die Vernetzung aller Akteure wünschenswert ist, um kommunale
Angebote für die Patienten attraktiver gestalten zu können. Insbesondere zur Untersuchung der Gestaltung und Umsetzung von Gemeindeangeboten besteht weiterhin großer Forschungsbedarf.

Strittig ist bisher, ob Prävention und Gesundheitsförderung zur Kosteneinsparung im Gesundheitswesen führen. Auf der Basis von DRG-Daten des Landesverbandes der Betriebskrankenkassen Niedersachsen-Bremen und Daten einer Wiederholungsbefragung von Teilnehmerinnen am BabyCare-Programm wurde von Kirschner, Halle und Pogonke die Inanspruchnahme medizinisch stationärer Leistungen vor, während und nach der Geburt analysiert und die entsprechenden Kosten berechnet. Das wichtige Fazit der Studie ist, dass mit wirksamen Programmen zur Verringerung von Frühgeburten wie dem BabyCare-Programm in Deutschland bei den gegebenen Geburten- und Frühgeburtenzahlen jährlich theoretisch 129 Mio. $€$ eingespart werden könnten. In Bezug auf Frühgeburten ist daher ein erhebliches Potenzial für Prävention und Kostensenkung gegeben.

Der möglichst frühzeitigen Prävention von Rückenleiden Rechnung tragend, ist das Modell „Rücken Kult-Tour“ zu nennen, welches Grundschulkinder, als auch Eltern und Lehrer einbezieht. Anhand einer Kontrollgruppen basierten Studie belegen Groll, Heinke-Goldammer, Zalpour positive Effekte hinsichtlich des Verhaltens und des Wissens über die Wirbelsäule und rückenschonender Verhaltensweisen, durch die Teilnahme an diesem innovativen Angebot.

Die Ergebnisse der Evaluationsstudie zur Nichtraucherkampagne „Nichtrauchen macht reich!“ an österreichischen Schulen zeigt, dass bei entsprechender ju- 
gendadäquater Gestaltung von Präventionsmaßnahmen, die an der Lebenswelt der Jugendlichen ansetzen, Jugendliche gut erreicht werden können. So zeigt insbesondere die gute Annahme der Website http://www.tabakfrei.at, dass die Kampagne von den Jugendlichen wahrgenommen wurde, entsprechende Wirkungen, wie Nachdenkprozess, Bewusstseinsbildung, Sensibilisierung, reduziertes Rauchverhalten etc. sehen die Autoren Neudorfer und Kvas als Effekte der Kampagne. Ebenfalls dem Thema Gesundheitsförderung junger Menschen widmet sich der Beitrag von Brunner. Wie ist es mit dem Kohärenzgefühl von jungen Menschen an der Universität bestellt? Hier zeigt die Messung des studentischen Kohärenzgefühls an einer österreichischen Universität, dass die Gefühle der Bewältigbarkeit, Handhabbarkeit und Sinnhaftigkeit mit der Einschätzung der subjektiven Lebensqualität verbunden sind.

Mit dem Thema der betrieblichen Gesundheitsförderung befassen sich drei Artikel. Die Autoren Beck und Lenhardt arbeiten heraus, dass die Gefährdungsbeurteilung abhängig ist von der Größe, dem Wirtschaftsbereich und von der wirtschaftlichen Lage der Betriebe. So zeigen die Ergebnisse der Erwerbstätigenbefragung, dass insbesondere Kleinst- und Kleinunternehmer hinsichtlich des Arbeits- und Gesundheitsschutzes erheblichen Beratungs- und Unterstützungsbedarf haben. Zielgruppenorientierung ist inzwischen für die Gesundheitsförderung selbstverständlich. Dennoch wird die Mitarbeiterperspektive in der betrieblichen Gesundheitsförderung bisher wenig berücksichtigt. Wie Mitarbeiter hinsichtlich der Informationsvermittlung und Angebotsgestaltung stärker berücksichtigt werden können, arbeitet die qualitative, auf der Auswertung von problemzentrierten Interviews mit verschiedenen Akteuren und Experten beruhenden Studie von Nöhammer, Eitzinger, Schaffenrath-Resi, Stummer heraus. So können Nutzungshemmnisse der betrieblichen Gesundheitsförderung reduziert werden. Abschließend fasst der Beitrag von Goldgruber und Ahrens die Ergebnisse aktueller Reviews über Wirksamkeitsstudien der betrieblichen Gesundheitsförderung und Primärprävention zusammen. Um- fassende Programme, die verhaltens- und verhältnispräventive Elemente umfassen, erzielen demnach den größten Nutzen.

\section{Hite Ollebrecut}

\author{
H. Ohlbrecht
}

\section{Korrespondenzadresse \\ Dr. phil. H. Ohlbrecht Dipl.-Soz.}

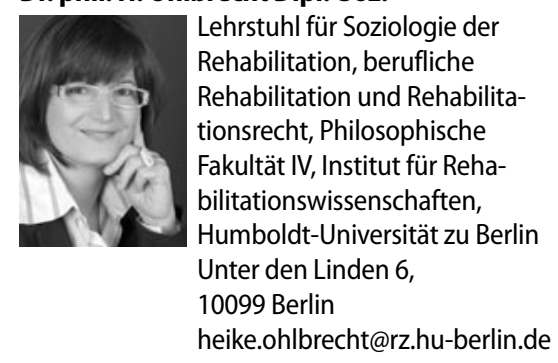

\section{Literatur}

1. Schiemann D, Blumberg P (2006) Entwicklung und Konsertierung des Expertenstandards Sturzprophylaxe in der Pflege. In: Deutsches Netzwerk für Qualitätsentwicklung in der Pflege (DNQP, Hrsg) Expertenstandards Sturzprophylaxe in der Pflege. Entwicklung Konsertierung Implementierung, Osnabrück 